\title{
Interacting Cold Rydberg Atoms: a Toy Many-Body System
}

\author{
Antoine Browaeys and Thierry Lahaye \\ Institut d'Optique \\ Laboratoire Charles Fabry \\ 2 av. A. Fresnel \\ 91127 Palaiseau cedex, France
}

\begin{abstract}
This article presents recent experiments where cold atoms excited to Rydberg states interact with each other. It describes the basic properties of Rydberg atoms and their interactions, with the emphasis on the Rydberg blockade mechanism. The paper details a few experimental demonstrations using two individual atoms or atomic ensembles, as well as applications of this "toy many-body system" to quantum information processing using photons.
\end{abstract}

\section{Introduction}

One of the great challenges in physics is to understand how complexity emerges from the simple underlying physical laws that govern the interactions between constituents of matter, like atoms or molecules. Interactions between particles are for the most part well-understood, and the equations of quantum mechanics governing an ensemble of interacting particles are known. However, due to the exponential scaling of the size of the Hilbert space with the number of particles, they are too difficult to be solved beyond a few tens of particles: this is called the many-body problem.

One idea to progress is to build in the laboratory some simple toy systems that are ruled by known but unsolved equations and to measure the properties of these systems. In doing so, one can validate or infirm theoretical approaches or models. Furthermore, by developing and harnessing quantum systems, one can envision technological developments. Presently, this quantum engineering has two main applications: quantum information processing and quantum metrology. Both rely on the ability to prepare particular quantum states of ensembles of particles exhibiting quantum correlations (also known as entanglement). These states can be prepared by controlling the interaction between the particles. Many physical systems are currently under investigation for quantum engineering: ions, atoms, photons, quantum circuits, electronic spins... The last decade has seen an explosion of theoretical and experimental studies of one such system, namely interacting cold Rydberg atoms.

Rydberg states are highly excited atomic states with a large principal quantum number $n$, close to the ionization threshold (for a detailed review, see [1]). Their study dates back to the early days of atomic spectroscopy. They are named after the Swedish physicist Johannes Rydberg who found in 1888 an empirical formula linking the various wavelengths of the spectral lines observed in alkali metals. Rydberg atoms played an important role in the birth of quantum mechanics; for instance 
Bohr's 1913 paper explicitly refers to the Rydberg formula [2]. They are an ideal playground to apply the correspondance principle. Rydberg atoms have exaggerated properties, and in particular exhibit very strong interactions.

This paper is organized as follows. After a brief reminder on the properties of Rydberg states and on their interaction, we will describe the concept of "Rydberg blockade" and review some important recent experiments studying the blockade. Finally we present two applications of this many-body system to quantum information processing using photons. A detailed review of the applications of Rydberg atoms to quantum information processing has been written by M. Saffman, T. Walker and K. Mølmer [3].

\section{Rydberg atoms and their interactions}

In this paper, we will concentrate on alkali atoms, which have one electron in their outer shell. All the experiments described here have been performed with heavy alkali atoms (rubidium or cesium).

\subsection{Rydberg atoms}

Classically, a Rydberg state corresponds to an orbit of the outer electron far from the nucleus. The electrons of the inner shells screen the charge of the nucleus and the atom is then hydrogenoid to a very good approximation (see fig. 1a). The energy of the atom in a Rydberg state with principal quantum number $n \gg 1$, orbital angular momentum $l$ and total angular momentum $j=l \pm 1 / 2$ is then

$$
E_{n, l}=-\frac{R_{y}}{n^{* 2}} \quad \text { with } \quad n^{*}=n-\delta_{l},
$$

and where $R_{y} \approx 13.6 \mathrm{eV}$ is the Rydberg constant. Here $\delta_{l}$ is the quantum defect that takes into account the fact that an electron with a low angular momentum $l$ can penetrate into the electronic core, thus increasing the effective charge seen by the electron and lowering the binding energy. Typically, for heavy alkali atoms, $\delta_{l \geq 3} \approx 0$.

Rydberg states exhibit interesting features, most of which can be understood with a semi-classical picture [1]. Among those, three are relevant to this paper:

- The dipole matrix elements $d=\left\langle n, l|\hat{D}| n^{\prime}, l^{\prime}\right\rangle$ coupling neighbouring Rydberg states $(n, l)$ and $\left(n^{\prime}, l \pm 1\right)$ are large: $d \approx n^{* 2} e a_{0}$ for $n^{*} \approx n^{* \prime}$ (e is the charge of the electron and $a_{0}$ the Bohr radius). As an exemple, for $n^{*}=50$, the transition dipole moment is 3000 times the dipole of a water molecule, already considered as a very polar object;

- As a consequence of this large dipole moment, the states are very polarizable, with the static polarizability $\alpha$ scaling as $n^{* 7}$. Rydberg atoms are therefore very sensitive to $\mathrm{DC}$ or AC electric fields. As an example, the state $58 d_{3 / 2}$ is shifted by $\sim 2.5 \mathrm{MHz}$ by a very small field of $0.1 \mathrm{~V} / \mathrm{cm}$;

- The states have very long radiative lifetime $\tau$, scaling like $\tau \propto n^{* 3}$. A lifetime of $\sim 100 \mu$ s for $n=50$ is typical. 


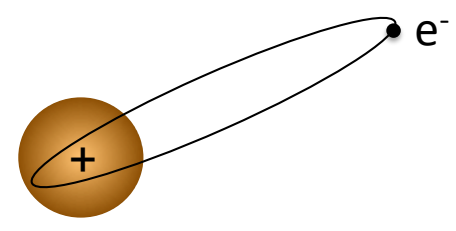

(a)

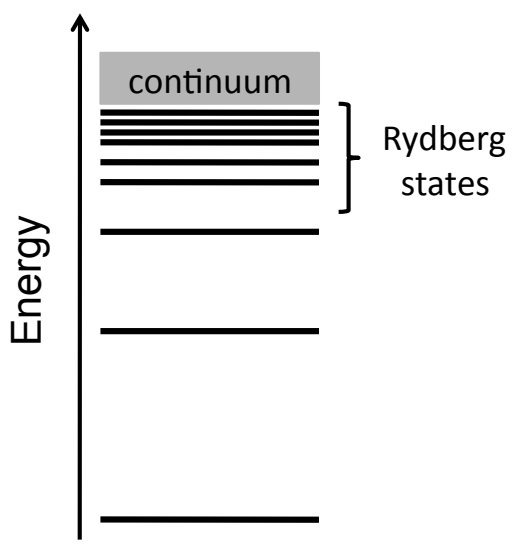

(b)

Figure 1: (a) Classical picture of an alkali Rydberg atom: the electron has an orbit far from the nucleus, which is screened by the electronic core. The resulting charge seen by the outer electron is therefore $+e$. When the outer electron has a low angular momentum, it penetrates the electronic core. (b) Quantum picture: Rydberg states are close to the ionization threshold. As a consequence, the energy spacing between Rydberg states decreases with increasing principal quantum numbers. Around $n=50$, for alkali atoms, the frequencies of the transitions between nearby states is in the $10-100 \mathrm{GHz}$ range.

\subsection{Interactions between Rydberg atoms}

The natural consequence of the large dipole moment featured by the Rydberg atoms is the large interaction between two of them. Let us consider two atoms $A$ and $B$ separated by a distance $R$ much larger than their size. The two atoms being neutral they interact predominantly via a dipole-dipole coupling, described by the hamiltonian:

$$
\hat{V}=\frac{1}{4 \pi \epsilon_{0} R^{3}}\left(\hat{\mathbf{d}}_{A} \cdot \hat{\mathbf{d}}_{B}-3\left(\hat{\mathbf{d}}_{A} \cdot \hat{\mathbf{r}}\right)\left(\hat{\mathbf{d}}_{B} \cdot \hat{\mathbf{r}}\right)\right)
$$

with $\hat{\mathbf{r}}=\mathbf{R} / R$ the unit vector joining the two atoms. This hamiltonian operates in the Hilbert space spanned by the two-atom basis $\left\{|n, l, j, m\rangle \otimes\left|n^{\prime}, l^{\prime}, j^{\prime}, m^{\prime}\right\rangle\right\}$.

Let us assume that the two atoms are in the $|n l\rangle$ state (in this discussion, we will drop the labels $j$ and $m$ ). Often the coupling by $\hat{V}$ of this state to other states is dominated by the coupling to one two-atom state, and the two-atom system can be reduced to an effective two-level system. For example, the difference in energy between the states $|n s, n s\rangle$ and $|n p,(n-1) p\rangle$ is usually much smaller than for all any other states $\left|n^{\prime} l^{\prime}, n^{\prime \prime} l^{\prime \prime}\right\rangle^{1}$. In this case the hamiltonian of the two atoms, reduced to the $|n s, n s\rangle$ and $|n p,(n-1) p\rangle$ basis is:

$$
\hat{H}=\left(\begin{array}{cc}
0 & C_{3} / R^{3} \\
C_{3} / R^{3} & \delta
\end{array}\right),
$$

with $\langle n s, n s|\hat{V}| n p,(n-1) p\rangle=C_{3} / R^{3}$ and $\delta=2 E_{n s}-E_{n p}-E_{(n-1) p}$. The eigen-

\footnotetext{
${ }^{1}$ In fact, one has a coupling to the two degenerate states $|n p,(n-1) p\rangle$ and $|(n-1) p, n p\rangle$. This is easily taken into account and does not change the conclusions of the qualitative discussion given here.
} 
energies of the two-atom system are

$$
\Delta E_{ \pm}=\frac{\delta}{2} \pm \frac{1}{2} \sqrt{\delta^{2}+\left(\frac{C_{3}}{R^{3}}\right)^{2}} .
$$

The interaction energy shows different forms depending on the distance between the atoms. If $C_{3} / R^{3} \ll \delta$, the state $|n s, n s\rangle$ is hardly admixed with the $|n p,(n-1) p\rangle$ state and its energy is

$$
\Delta E_{n s, n s} \approx \frac{1}{2 \delta}\left(\frac{C_{3}}{R^{3}}\right)^{2} .
$$

This is the van der Waals limit where the interaction energy scales as $C_{6} / R^{6}$. In this regime the only effect of the interaction is to shift the state $|n s, n s\rangle$ by an amount $\Delta E_{n s, n s}$. A simple scaling argument shows that $C_{6} \propto n^{* 11}$. Indeed $C_{3} \propto d^{2} \propto n^{* 4}$. Furthermore, according to Eq. (1), one has $\delta \propto 1 / n^{* 3}$, yielding the $n^{* 11}$ scaling. In practice, several states can have contributions of the same order of magnitude; in this case the van der Waals shift of the two-atom states $|n l, n l\rangle$ induced by the dipole-dipole interaction can be calculated by means of second-order perturbation theory (see e.g. [4]):

$$
\Delta E_{n l, n l}=\sum_{n^{\prime}, l^{\prime}, n^{\prime \prime}, l^{\prime \prime}} \frac{\left|\left\langle n l, n l|\hat{V}| n^{\prime} l^{\prime} n^{\prime \prime} l^{\prime \prime}\right\rangle\right|^{2}}{2 E_{n l}-E_{n^{\prime} l^{\prime}}-E_{n^{\prime \prime} l^{\prime \prime}}} .
$$

If on the contrary the atoms are so close that $C_{3} / R^{3} \gg \delta$, the two eigen-energies of the hamiltonian are

$$
\Delta E_{ \pm} \approx \pm \frac{C_{3}}{R^{3}}
$$

corresponding to the states $| \pm\rangle=\frac{1}{\sqrt{2}}(|n s, n s\rangle \mp|n p,(n-1) p\rangle$.

At a given distance it is possible to tune from one regime to another by taking advantage of the DC-Stark shift. Provided the fact that $\delta$ is already small, a DC field shifts the two states $|n s, n s\rangle$ and $|n p,(n-1) p\rangle$ relatively to each other, making it possible to achieve $\delta=0$. In this case the interaction energy scales as $1 / R^{3}$ whatever the distance between the two atoms. This situation is called a Förster resonance. In the case of rubidium it is easy to achieve resonance with very weak electric fields around the states $43 d_{5 / 2}$ and $59 d_{3 / 2}$, but many other combinations also exist.

To summarize, the interaction energy between two Rydberg atoms exhibits a strong dependency with the principal quantum number $n^{*}$, and can be tuned from the van der Waals regime to the $1 / R^{3}$ regime using an electric field or by decreasing the distance between the two atoms.

Figure 2(a) presents the result of an ab-initio calculation of the interaction energy between two rubidium atoms in the $62 d_{3 / 2}$ states. This graph shows that the interaction energy is as large as a $10 \mathrm{MHz}$ for a separation of $6 \mu \mathrm{m}$. For the same separation the interaction energy between the atoms in their ground state would be $\sim 10^{-4} \mathrm{~Hz}$. These values are typical for alkali atoms and show that the strength of the interaction can be enhanced by 11 orders of magnitude by exciting the atoms to a Rydberg state. Finally, this interaction is switchable as the atoms can be excited by lasers for a given duration. 
(a)

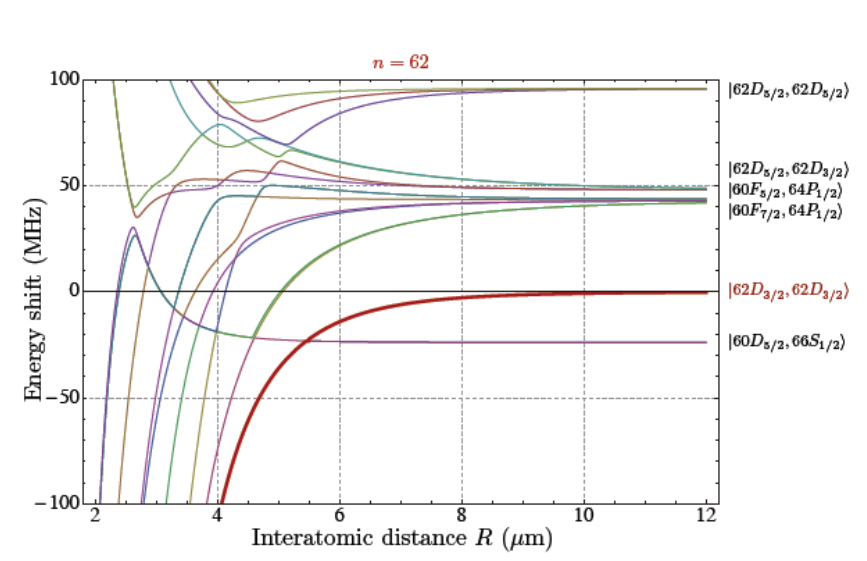

(b)

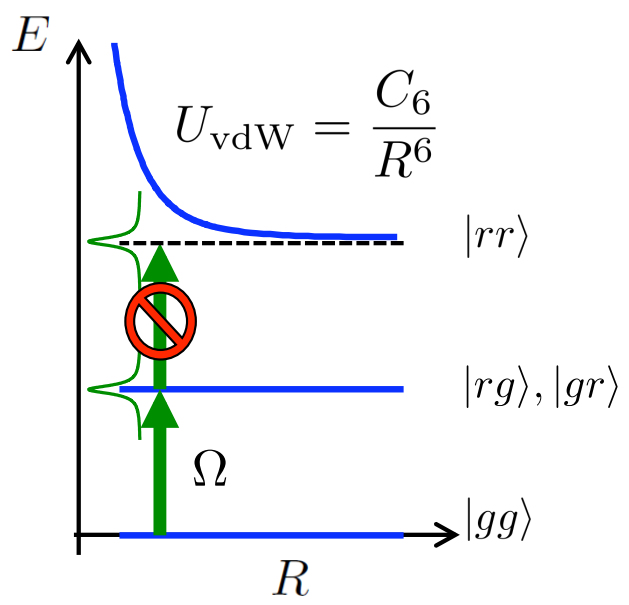

Figure 2: (a) Potential curves corresponding to the interaction energy between two rubidium 87 atoms around the Rydberg states $62 d_{3 / 2}$ (courtesy Lucas Béguin). (b) Principle of the Rydberg blockade between two atoms in the van der Waals regime.

\subsection{Early experiments}

One of the first observation of the interaction between Rydberg atoms was reported in 1981 by J.-M. Raimond and co-workers [5]. The authors used a dense thermal beam of cesium (density $\geq 10^{13}$ at $/ \mathrm{cm}^{3}$ ) at $300 \mathrm{~K}$ and excited the atoms to the Rydberg states with 10 ns-pulsed laser. They observed a broadening of the line that they attributed to the van der Waals interaction between the Rydberg atoms.

This first demonstration was achieved using atoms at room temperature. When laser cooled and trapped atomic samples became available, the study of interacting Rydberg gases was facilitated. For these samples, with temperature lower than $100 \mu \mathrm{K}$, the interaction energy between two Rydberg atoms overcomes their kinetic energy for the range of spatial densities achievable in the lab $\left(10^{10}-10^{13} \mathrm{at} / \mathrm{cm}^{3}\right)$. In this regime of "frozen gases", the behavior of the gas is mainly governed by the interactions and many-body effects are dominant.

Two pioneering experiments were performed simultaneously in 1998, which started the field of the study of strongly interacting cold atomic samples. In these two experiments, one performed in laboratoire Aimé Cotton (Orsay, France) [6] and the other at the university of Virginia (USA) [7], the experimentalists excited a gas of laser cooled and trapped atom at a Förster resonance. They observed that the width of the spectral line was much larger than what is expected from a model where interactions are taken into account by a mean-field approach. In this model the width of the line is expected to be $C_{3} / \bar{R}^{3}$, with $\bar{R}=\rho^{-1 / 3}$ and $\rho$ the density of the gas. However, the observed width was 2 orders of magnitude larger than this prediction. The authors proposed a model including the diffusion of the Rydberg excitation through the gas. For example, for the case or the Aimé Cotton experiment, the authors used the Förster resonance $23 p_{3 / 2}+23 p_{3 / 2} \rightarrow 23 s+24 s$. The atoms are excited in the $p$ states and therefore the dipole-dipole interaction rapidly transfers closely spaced atoms to $s$ states. Another resonance, always present, $s+p \rightarrow s+p$ ensures that, on slower timescales, the $s$ excitation can migrate through the gas, leading to new transfers. In this way, all the atoms participate to the behavior of 
this many-body system.

\section{Rydberg blockade and many-body physics: theoretical aspects}

Following the two demonstrations that Rydberg interactions between cold atoms could lead to interesting many-body effects, two theoretical works proposed to use the interaction between two cold Rydberg atoms to generate entangled states $[8,9]$. Both proposals rely on the "Rybderg blockade", which we now describe.

Consider two atoms $A$ and $B$, separated by a distance $R$. Each atom is supposed to behave as a two-level system with ground state $|g\rangle$ and Rydberg state $|r\rangle$, separated by an energy $E$. The spectrum of the two-atom system consists in 4 states $|g g\rangle,|g r\rangle,|r g\rangle$ and $|r r\rangle$ (see Fig. 2b). Assuming that the atoms are in the van der Waals regime, the state $|r r\rangle$ is shifted by an amount $U_{\mathrm{vdW}}$, all the other states being essentially unshifted, as the atoms only interact strongly when they are both in the Rydberg state. As a consequence, a laser, tuned on resonance with the excitation of one atom, is not resonant with the excitation of the second atom, provided that the linewidth of the excitation is smaller than $U_{\mathrm{vdW}}$. This is the so-called "Rydberg blockade": the excitation of one atom prevents the excitation of the second one.

When the two atoms are excited by the same laser field with electric field $E_{\mathrm{L}}$, a consequence of the blockade is the preparation of a superposition of having excited one or the other atom. The state prepared in this way is entangled and reads

$$
\left|\psi_{+}\right\rangle=\frac{1}{\sqrt{2}}(|r g\rangle+|g r\rangle)
$$

Importantly, when prepared in state $\left|\psi_{+}\right\rangle$, the two atoms behave collectively with respect to the excitation. This means that the total dipole of the two atoms is $\left\langle g g\left|\hat{D}_{A}+\hat{D}_{B}\right| \psi_{+}\right\rangle=\sqrt{2} d$, with $d=\left\langle g\left|\hat{D}_{A}\right| r\right\rangle=\left\langle g\left|\hat{D}_{B}\right| r\right\rangle$. Note that the antisymmetric state $\left|\psi_{-}\right\rangle=(|r g\rangle-|g r\rangle) / \sqrt{2}$ is not coupled to the laser, as the total dipole is 0 . When in the Rydberg blockade regime, the two-atom system will undergo Rabi oscillations between the collective states $|g g\rangle$ and $\left|\psi_{+}\right\rangle$at a frequency $\sqrt{2} \Omega$, where the single-atom Rabi frequency $\Omega=d E_{\mathrm{L}} / \hbar$ characterizes the coupling between the states $|g\rangle$ and $|r\rangle$.

The blockade of the excitation occurs when the atoms are within a blockaded sphere of radius $R_{\mathrm{b}}$, which is set by the bandwidth of the excitation. Therefore, in the case of the van der Waals interaction:

$$
\hbar \Omega=\frac{C_{6}}{R_{\mathrm{b}}^{6}} \Rightarrow R_{\mathrm{b}}=\left(\frac{\hbar \Omega}{C_{6}}\right)^{1 / 6}
$$

As an example for the state $62 d_{3 / 2}$ of rubidium and for $\Omega / 2 \pi=1 \mathrm{MHz}, R_{\mathrm{b}} \approx 10 \mu \mathrm{m}$. When the bandwidth of the excitation is set by the linewidth $\Delta \nu$ of the laser, the blockade radius is obtained by replacing the Rabi frequency $\Omega$ by $\Delta \nu$.

The Rydberg blockade can be extended to the case of $N$ atoms all located within the blockade sphere. Then the $N$-atom system will oscillate between the collective state $|g g g \ldots g\rangle$ and

$$
\left|\psi_{\mathrm{c}}\right\rangle=\frac{1}{\sqrt{N}} \sum_{j=1}^{N}\left|g g \ldots r_{j} \ldots g\right\rangle
$$


where only one of the $N$ atoms is excited to the state $|r\rangle$. The frequency of the oscillation is then $\sqrt{N} \Omega$, with $\Omega$ the single atom Rabi frequency. This collective oscillation emphasizes the many-body character of the system. The state $\left|\psi_{\mathrm{c}}\right\rangle$ is sometimes called a polariton. It corresponds to an atomic excitation delocalized over the entire ensemble.

\section{Observation of the Rydberg blockade and collective excitation of two atoms}

After the publication of the pioneering theoretical proposals [8, 9], it took another 8 years before the blockade between two individual atoms was demonstrated in the lab. This section summarizes the first two demonstrations of the blockade and the implications for the preparation of entangled states and the demonstration of quantum gates.

\subsection{Individual atoms in optical dipole traps}

Techniques for laser cooling and trapping of neutral atoms by laser light were developed in the 80's and 90's. It was in particular demonstrated that atomic samples could be cooled down to temperatures below $100 \mu \mathrm{K}$ starting from vapors at room temperature. The basic mechanism that makes laser cooling possible is the radiation pressure force that the light exerts on the atoms (for a review of this field, see e.g. $[10,11,12])$.

It is also possible to use a non dissipative atom-light interaction to produce conservative traps in which cold atoms can be stored. The principle is the following. The electric field $E_{\mathrm{L}}$ of a laser at frequency $\omega$ induces an atomic dipole $d=\alpha E_{\mathrm{L}}$. Here $\alpha$ is the polarizability of the atom. In the case of a laser detuned with respect to an atomic transition at frequency $\omega_{0}, \alpha \propto 1 /\left(\omega_{0}-\omega\right)$. The dipole interacts with the laser field giving rise to an interaction energy $U=-\left\langle d E_{\mathrm{L}} / 2\right\rangle=-\alpha E_{\mathrm{L}}^{2} / 2$, where $\langle$.$\rangle denotes a time average over many oscillations of the laser field. Therefore, when$ the laser is red-detuned with respect to the atomic transition $\left(\omega-\omega_{0}<0\right)$, the dipole and the field are in phase and a low energy is obtained for a large amplitude of the electric field. Close to the waist of a gaussian laser beam, the field is the largest at the position of the focus, and decreases away from it. This configuration of a focused beam therefore constitues a trap. Easily available laser powers lead to trap depths $U / k_{\mathrm{B}}=0.1-1 \mathrm{mK}$. This kind of trap is called a dipole trap.

In the late 90 's and early 2000, it was demonstrated that it is possible to extend the techniques of dipole trapping in a regime where only one atom is confined. This configuration relies on a microscopic dipole trap, which is produced by focusing a laser beam onto a spot of size $w \approx 1 \mu \mathrm{m}$ using a large numerical aperture lens. It was demonstrated for the first time at the Institut d'Optique in $2001[13,14]$. The microscopic trap is focused inside the cloud of a magneto-optical trap (MOT), the MOT cooling laser being on during the whole loading procedure. The loading of the microscopic trap results from the competition between two processes: (i) an atom of the magneto-optical trap enters in the dipole trap and is slowed down thanks to the cooling lasers of the MOT; (ii) as soon as a second atom enters the dipole trap, a two-body inelastic collision catalyzed by the light results in the rapid loss of the two atoms. This loss is fast due to the microscopic volume of the trap. The loss prevents the simultaneous presence of two trapped atoms, as long as the loss rate is 


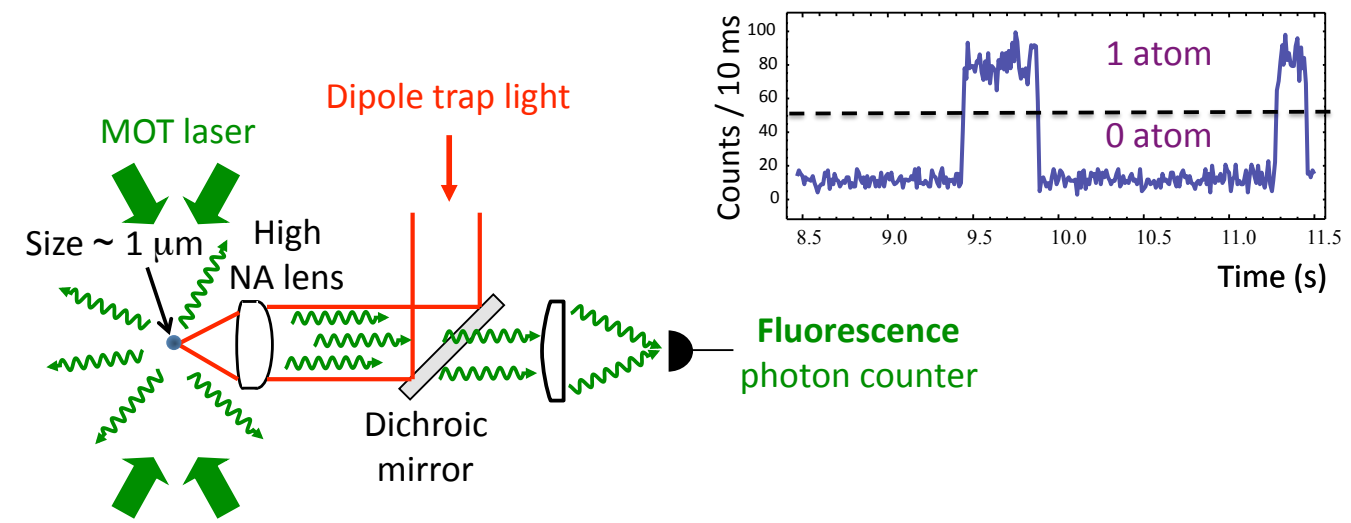

Figure 3: Trapping of an individual atom in a microscopic dipole trap. The dipole trap beam is focused, by a high numerical aperture (NA) lens, inside a MOT (not shown). The fluorescence light induced by the MOT beams is collected by the same lens, separated from the dipole trap light by a dichroic mirror and sent onto a photon counter. An example of fluorescence signal as a function of time is shown in the inset.

faster than the loading rate. Figure 3 shows a typical trapping configuration, with the microscopic trap and the imaging system to collect the fluorescence from the trap region. This fluorescence, induced by the MOT laser beams, is collected onto a single-photon counter. The figure also shows an example of fluorescence signal as a function of time. The signal toggles at random between periods of low values corresponding to an empty trap, and periods of high value reflecting the presence of an atom. The absence of double steps is the signature that at most only one atom is present in the trap. It is therefore possible to trigger an experiment on the presence of an atom in the trap, thus realizing an heralded, but non deterministic, single atom source. This procedure can be extended to a larger number of traps, although in this case one has to wait until all the traps are filled at the same time. The probability of simultaneous occupancy then decreases exponentially with the number of traps.

\subsection{Observation of the blockade: the University of Wisconsin experiment [15]}

In this experiment, the group at the University of Wisconsin (USA) trapped two cesium atoms in two dipole traps separated by a distance of $R \approx 10 \mu \mathrm{m}$, see figure 4(a). Here, each atom can be excited separately, one atom being considered as a control atom, the other one as the target atom. After preparing the atoms in one of the hyperfine ground state $|g\rangle$, the experimentalists first excited the target atom to the Rydberg state $|r\rangle$ using a two-photon transition (an example of two-photon transition is shown in figure 5a). As they vary the duration of the excitation, they observed the characteristic Rabi oscillations between the states $|g\rangle$ and $|r\rangle$.

In order to demonstrate the blockade, the authors started by exciting the control atom to the Rydberg state by applying a $\pi$ pulse. They then sent the excitation laser on the target atom and observed that the probability to excite it to the Rydberg state was strongly suppressed as shown in figure 4 . This is the signature of the Rydberg blockade and the proof that the Rydberg excitation of the target atom is controlled by the state of the control atom. 

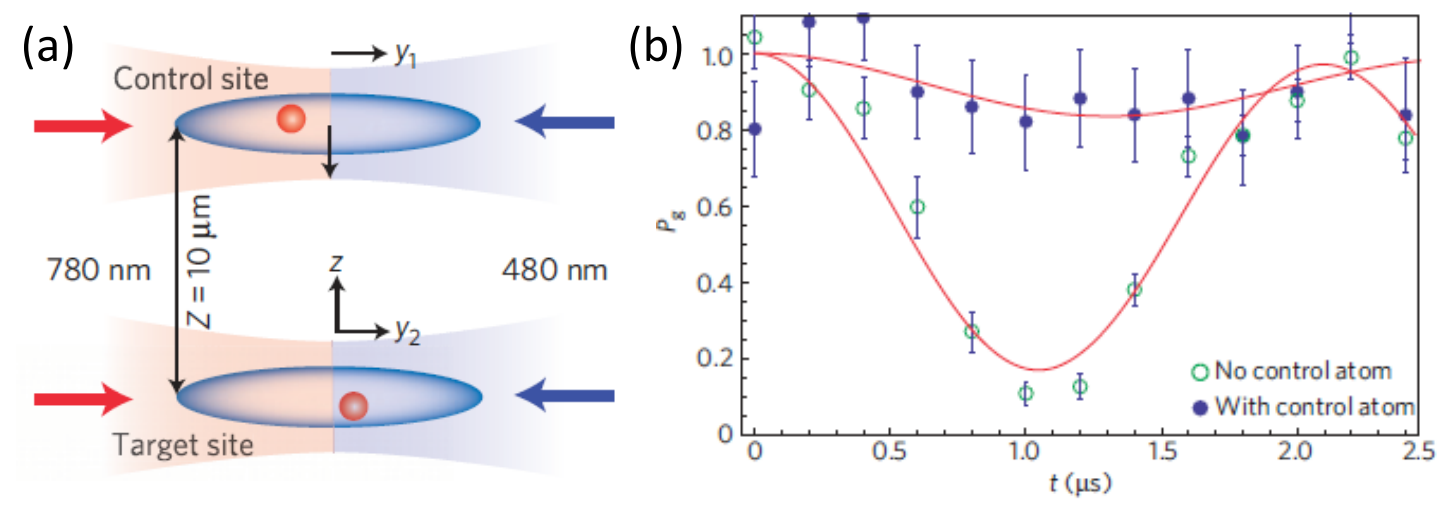

Figure 4: Observation of the blockade between two atoms. (a) The two traps are separated by $R=10 \mu \mathrm{m}$ and the two atoms can be excited separately from each other by independent laser beams. (b) Rabi oscillation on the target atom without or with the control atom in the Rydberg state. Each data point is an average over many realizations of the experiment in order to measure the probabilities. Figures from [15].

A year after this demonstration, the same group used the blockade to demonstrate a C-NOT quantum gate, using the protocol from the original proposal [8]. This quantum gate involves two hyperfine ground states of the cesium atom, labeled $|0\rangle$ and $|1\rangle=|g\rangle$, and the Rydberg state $|r\rangle$ as an intermediate state in the sequence. Its table of truth is the following one, with the first and second quantum bits corresponding to the control and target atoms respectively:

$$
\begin{aligned}
& |00\rangle \rightarrow|00\rangle \\
& |01\rangle \rightarrow|01\rangle \\
& |10\rangle \rightarrow|11\rangle \\
& |11\rangle \rightarrow|10\rangle .
\end{aligned}
$$

The Rydberg blockade is the underlying mechanism, which allows or not the flipping of the state of the target atom depending on the state of the control atom. The Wisconsin group reported an experimental fidelity of the gate of 0.73 [16]. They also used the gate to demonstrate the preparation of entangled states. The C-NOT gate is indeed an entangling gate: if the control atom is prepared in the superposition $(|0\rangle+|1\rangle) / \sqrt{2}$, and the target state in $|0\rangle$, the action of the gate leads to the final two-atom state $(|00\rangle+|11\rangle) / \sqrt{2}$. The fidelity of the entangled states was reported to be around 0.6 .

\subsection{Observation of the collective excitation: the Institut d'Optique experiment [17]}

Our group at Institut d'Optique demonstrated the blockade in a complementary experiment. There, the two atoms $A$ and $B$ were trapped in two dipole traps separated by a distance of $4 \mu \mathrm{m}$, see figure $5(\mathrm{a})$. In this experiment, the Rydberg excitation laser does not address a specific atom. The experiment then goes as follows. We first performed a Rabi oscillation with only atom $A$ present, the second trap being empty. We observed the Rabi oscillations between state $|g\rangle$ and $|r\rangle$, as shown in figure $5(\mathrm{~b})$. The experiment is then repeated when the two traps contain one atom each. We measured the probability to excite the two atoms at the same time $P_{r r}$ 


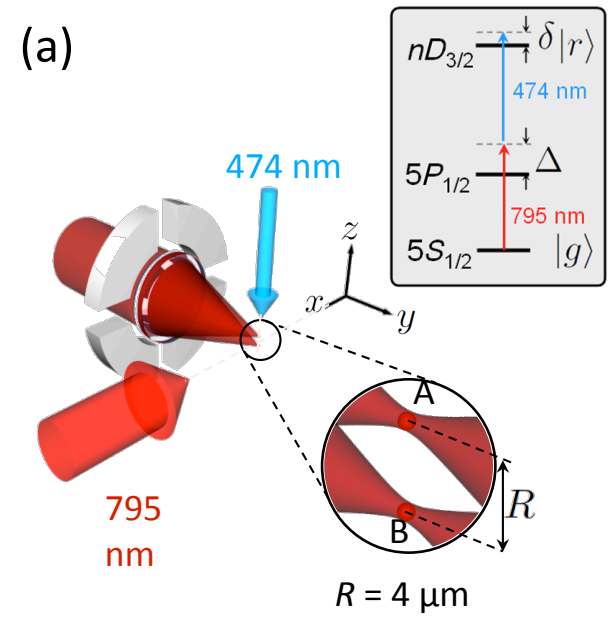

(b)

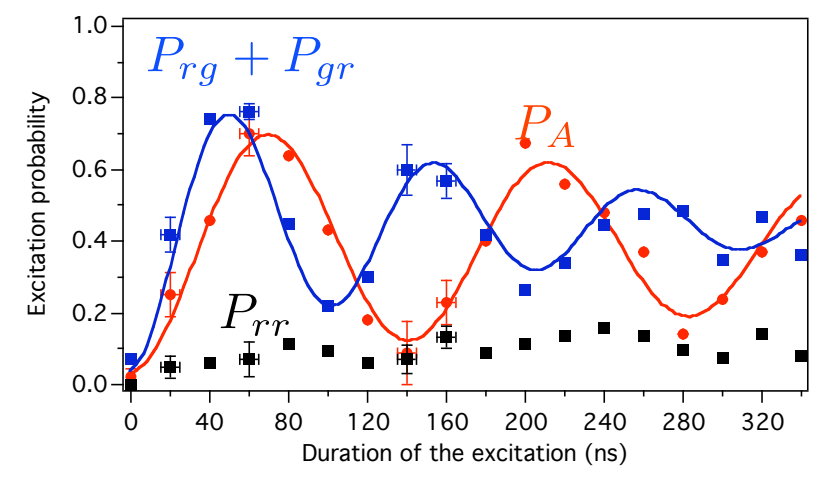

Figure 5: Observation of the blockade and of the collective excitation of two atoms. (a) Here the two traps are separated by $4 \mu \mathrm{m}$. The excitation lasers do not address the atoms independently, as their size is much larger than the interatomic distance. The atoms are excited by a two-photon transition. (b) $P_{A}$ : probability to excite atom A alone when the trap B is empty. $P_{r r}$ : probability to excite the two atoms at the same time. $P_{r g}+P_{g r}$ : probability to excite one of the two atoms. Each data point is an average of 100 realizations of the experiment, in order to calculate the probabilities. Figures from $[17,19]$.

and the probability to excite only one of the two atoms $P_{r g}+P_{g r}$. The results are shown in figure 5(b). The probability of exciting the two atoms at the same time is indeed suppressed, as it should be for two atoms in the blockade regime. However, the probability to excite one of the two atoms does oscillate, and the oscillation frequency appears faster than when only one atom is present. The ratio of the two measured Rabi frequencies is 1.38 , in very good agreement with the theoretical prediction $\sqrt{2}$. This enhancement of the oscillation frequency is the signature of the collective excitation of the two atoms: in the blockade regime, the laser couples the two collective states $|g g\rangle$ and $\left|\psi_{+}\right\rangle=(|r g\rangle+|g r\rangle) / \sqrt{2}$.

The collective state $\left|\psi_{+}\right\rangle$prepared in this way has the drawback that it involves one atom in the Rydberg state, which is not trapped in the dipole trap, and is relatively short-lived. We therefore mapped the Rydberg state to a second hyperfine ground state $\left|g^{\prime}\right\rangle$, which is trapped and long-lived. In this way, we prepared the entangled Bell state $\left(\left|g^{\prime} g\right\rangle+\left|g g^{\prime}\right\rangle\right) / \sqrt{2}$, with a fidelity of 0.75 [18].

\subsection{Measurement of the interaction energy between two atoms [19]}

It is interesting to investigate the dynamics of the two atoms when they are in an intermediate regime such that $\hbar \Omega \approx C_{6} / R^{6}$. There the dynamics does not only depend on the Rabi frequency alone, but also on the interaction energy between the two atoms. Our group at Institut d'Optique measured the probability $P_{r r}(\tau)$ to excite the two atoms at the same time in this "partial blockade" regime, as a function of the duration $\tau$ of the excitation. An example of data is shown in figure 6(b) and presents a non-trivial dynamics. A fit to the data by a simple model taking into account only the three states shown in figure 6(a) yields the interaction energy, when the Rabi frequency is measured independently using only one atom. We could 

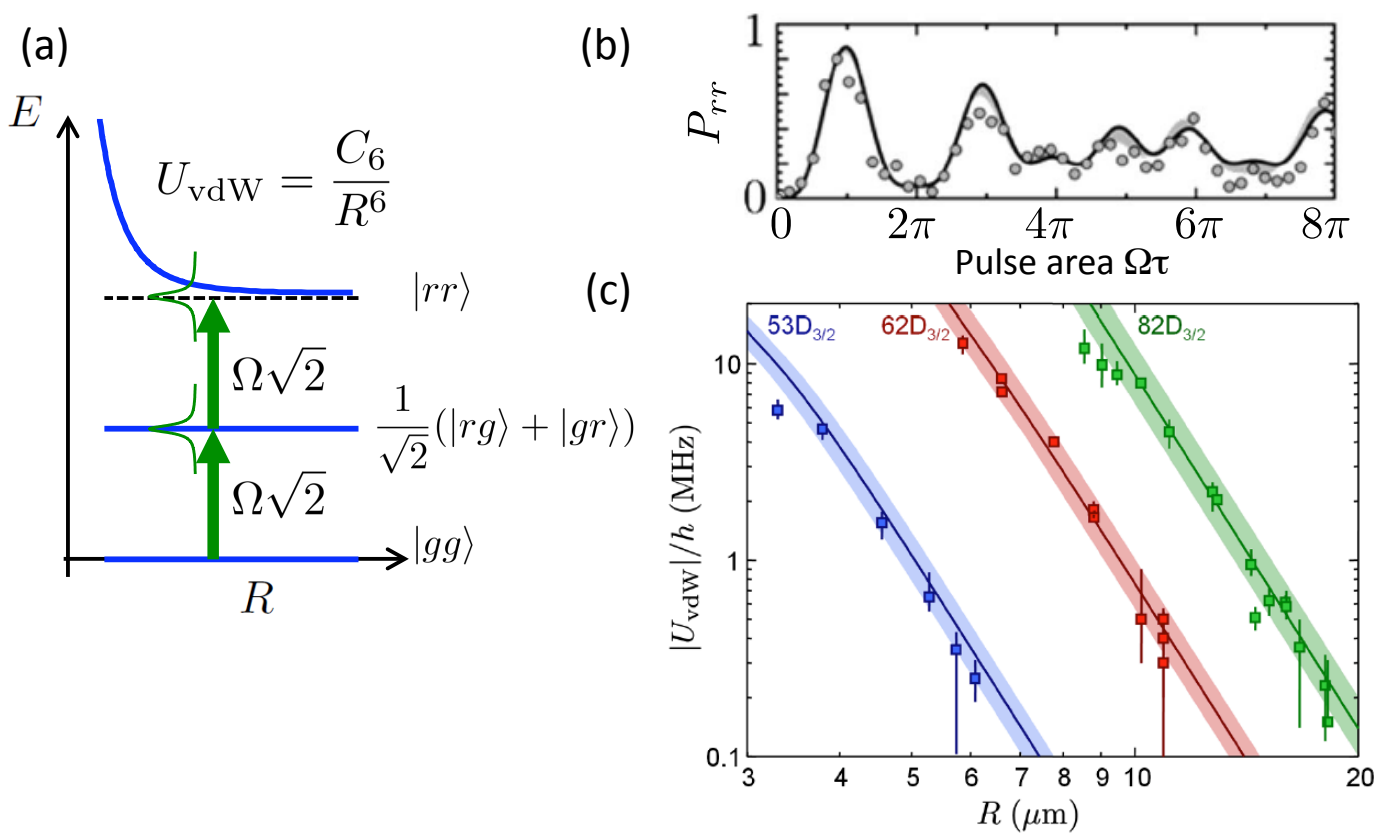

Figure 6: (a) Partial blockade regime where $\hbar \Omega \approx C_{6} / R^{6}$. (b) Probability of exciting the two atoms at the same time as a function of the pulse area $\Omega \tau$. Here $\Omega$ is the single atom Rabi frequency and $\tau$ the duration of the pulse. The distance between the atoms is $R=10 \mu \mathrm{m}$ and $\Omega=2 \pi \times 0.9 \mathrm{MHz}$. (c) Interaction energy between the atoms deduced from a fit of oscillations such as the one in (b), as a function of their separation. Figure from [19].

then measure directly the van der Waals interaction between two atoms as a function of their separation $R$. The results of the measurement are shown in figure 6(c) for three different Rydberg states, together with the result of an ab-initio calculation of the interaction energy. The data highlights the dramatic scaling of the van der Waals energy with the principal quantum number $n$ : at a given distance, changing $n$ from 58 to 62 increases the interaction energy by a factor of about 50 .

\section{Rydberg blockade in atomic ensembles}

In parallel to the work on the manipulation of a few individual atoms, the Rydberg blockade was also investigated in large cold atomic ensembles. In this case, the atoms are randomly distributed in space.

\subsection{Rydberg blockade in cold atomic clouds}

The first demonstration of the Rydberg blockade in a gas of cold atoms was performed in 2004 by a group at the university of Connecticut (USA) [20]. This group used a magneto-optical trap of rubidium atoms as the atomic ensemble and excited the atoms to the Rydberg states by a pulsed laser at a wavelength $297 \mathrm{~nm}$. The experimentalists measured the number of Rydberg atoms produced as a function of the intensity of the excitation laser for different principal quantum number $n$. The excitation being incoherent, they expected the number of Rydberg atoms produced to be proportional to the intensity of the laser, in the absence of interaction. The 


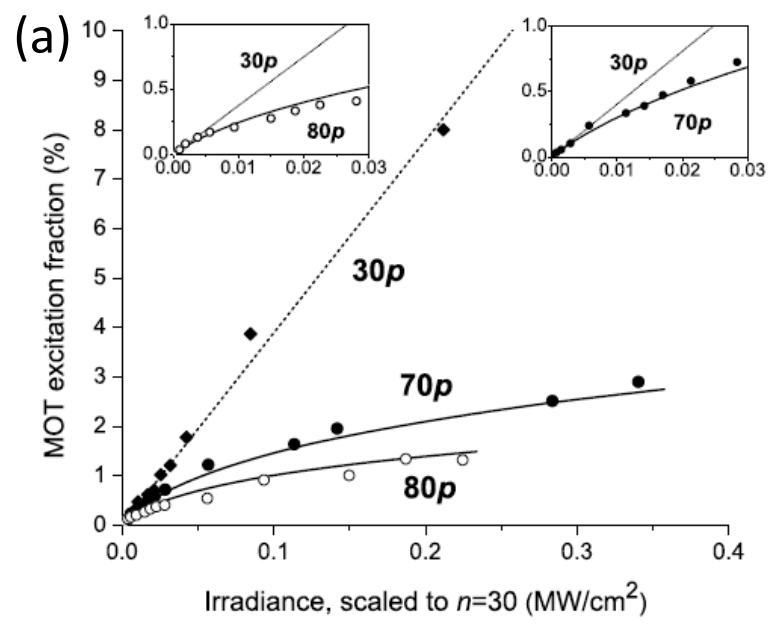

(b)

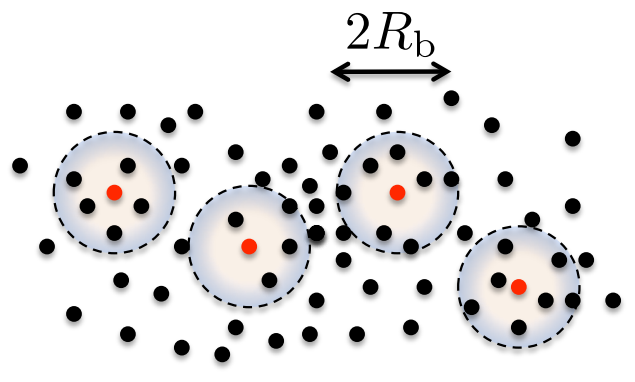

Figure 7: (a) Demonstration of the Rydberg blockade in the cloud of a magneto-optical trap. The data show the fraction of atoms excited to various Rydberg states as a function of the intensity of the pulsed excitation laser. The suppression of the number of Rydberg atoms for high principal quantum numbers is the signature of the blockade. From [20]. (b) Blockade sphere picture of the Rydberg blockade in an atomic ensemble with random positions of the atoms. Around each Rydberg atoms (in red), the excitation of neighboring atoms is impossible.

observations are reported in figure 7(a). They show a suppression of the number of Rydberg atoms produced when the frequency of the laser is tuned to states of increasing principal quantum numbers. As seen in Section 2.2, increasing the principal quantum number is equivalent to increasing the van der Waals interaction between the atoms. The experimental findings are in agreement with the concept of the blockade sphere introduced in Section 2.2: as soon as one atom is excited to the Rydberg state, it prevents the excitation of the atoms located within a sphere of radius $R_{\mathrm{b}}$, as represented in figure $7(\mathrm{~b})$. As a consequence, assuming that the atomic cloud is a sphere of radius $R$, the largest number of Rydberg atoms that can be produced is $\left(R / R_{\mathrm{b}}\right)^{3}$. The group at university of Connecticut used a MOT, resulting in a relatively low density of the cloud $\left(\leq 10^{11}\right.$ at $\left./ \mathrm{cm}^{3}\right)$. This prevented them from reaching the saturation predicted by the blockade sphere model. Furthermore, the group used an incoherent pulsed excitation, hampering the observation of the underlying collective Rabi oscillations. Soon after this first demonstration, several groups also observed the blockade in cold atomic samples in the van der Waals regime or using a Förtser resonance to enhance the interactions between the atoms, still in relatively dilute atomic clouds from magneto-optical traps. The review paper [21] gives an overview of these experiments.

In 2007, physicists at the university of Stuttgart repeated the experiment with a much denser atomic cloud $\left(\sim 10^{14} \mathrm{at} / \mathrm{cm}^{3}\right)$ and a coherent two-photon excitation with a well-controlled Rabi frequency $\Omega_{0}$ [22]. This allowed them to check some scaling laws that confirmed the idea of collective Rabi oscillation and blockade sphere. The measurements are shown in figure 8(a): the number of atoms excited to the Rydberg state increases linearly at short times and saturates afterwards. The authors explained this behavior as resulting from the incoherent sum of many collective Rabi oscillations at frequency $\Omega_{0} \sqrt{N}$ in each blockade sphere, with an inhomogeneous distribution of the number of atoms in the spheres across the cloud. This incoherent 
(a)

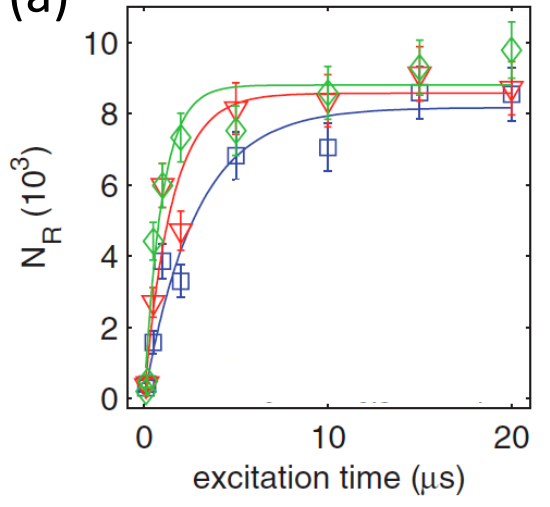

(c) a

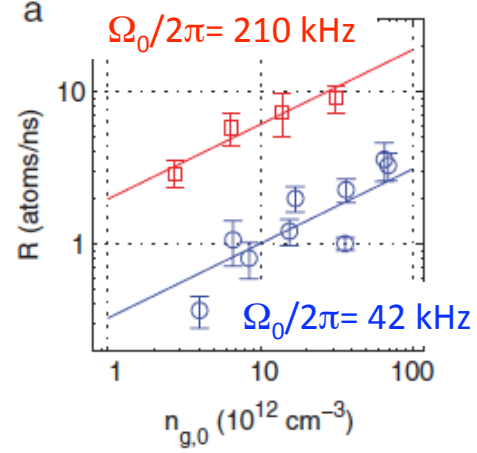

(b)
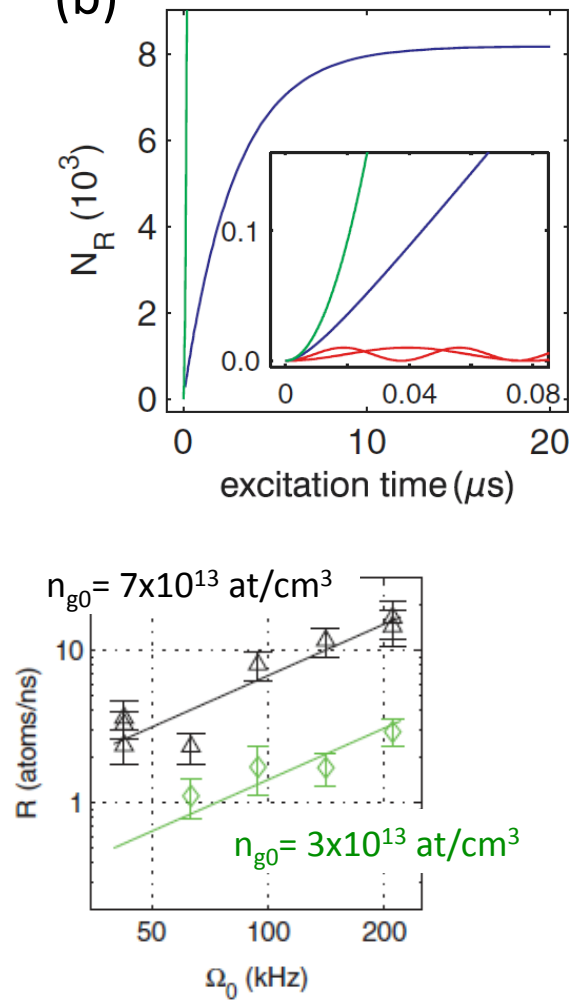

Figure 8: Verification of the scaling laws for the collective Rydberg excitation in the blockade regime. The figures are taken from [22]. (a) Evolution of the number of Rydberg atoms as a function of the excitation time for an increasing density of the cloud. (b) This evolution is reproduced by a model with an incoherent sum of Rabi oscillations $\sin ^{2}\left(\Omega_{0} \sqrt{N} t / 2\right)$ in each blockade sphere containing $N$ atoms, with an inhomogeneous distribution of the number of atoms across the cloud. (c) Demonstration of the scaling law $R \sim n_{g 0} \sqrt{\Omega_{0}}$ for the initial rate of formation of Rydberg atoms, as a function of the ground state density $n_{g 0}$ and the Rabi frequency $\Omega_{0}$.

sum leads to a linear increase of the number of Rydberg atoms at short time, followed by a saturation at later time, as represented in figure 8(b). The authors analyzed the variation of the initial slope $R$ with the density $n_{g 0}$ of the cloud and with the Rabi frequency $\Omega_{0}$ of the laser excitation. This slope should be proportional to $\Omega_{0} \sqrt{\langle N\rangle}$, with $\langle N\rangle \propto n_{g 0}$ the average number of atoms in a blockade sphere. Figure 8(c) shows that this is indeed the case, as a fit of the rate to a power law gives $R \propto n_{g 0}^{0.49} \Omega_{0}^{1.1}$. In a similar way, the authors showed that the maximal number of Rydberg atoms $N_{\mathrm{R}}$ is proportional to $1 / R_{\mathrm{b}}^{3} \propto \sqrt{\Omega_{0}}$ and independent on the density of ground state atoms $n_{g 0}$.

Finally, the improvement of experimental techniques led recently to the direct observation of the collective Rabi oscillation in an atomic sample with a size smaller than the blockade radius [23]. The authors started from a cold atomic ensemble in a one-dimensional optical lattice. The Rydberg excitation volume was determined by the size of the excitation laser beams at the position of the atoms. The resulting sphere had a radius of $\sim 15 \mu \mathrm{m}$. By choosing a principal quantum $n=102$ and a linewidth of the excitation $\sim 1 \mathrm{MHz}$, all the excitation volume was within a blockade 


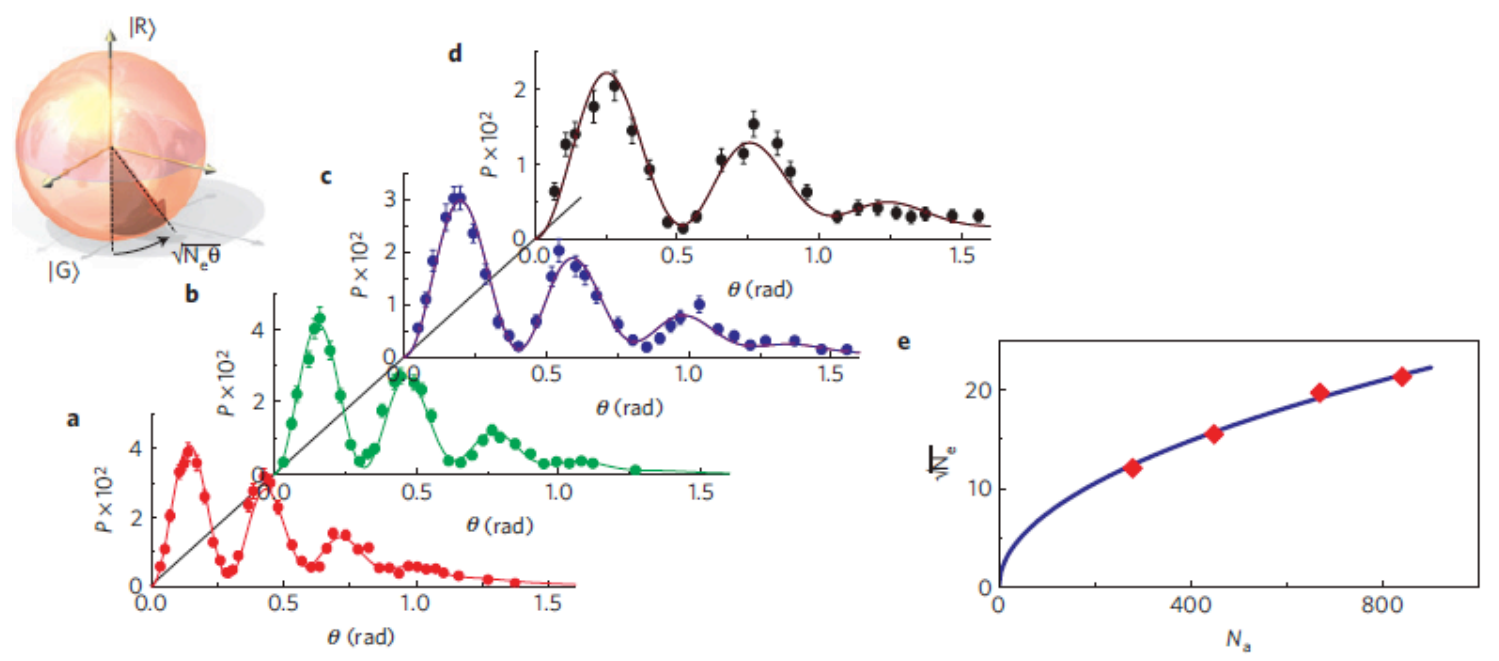

Figure 9: (a) Collective Rabi oscillations in an atomic sample with a size smaller than the blockade radius, from [23]. (a)-(d) Probability to excite only one atom in the ensemble for lowering atom numbers. (e) Collective Rabi frequency as a function of the number of atoms in the sample, together with a fit by a $\sqrt{N}$ law.

sphere. The authors then observed the collective Rabi oscillations, with the expected $\sqrt{N}$ scaling of the Rabi frequency when they varied the number $N$ of atoms in the volume (see figure 9).

\subsection{Spatially resolved observation of the blockade [24]}

The blockade sphere picture was further confirmed by an elegant experiment performed in Munich in 2012. The group there trapped atoms in a regular twodimensional array obtained by interfering laser beams (optical lattice), as represented in figure 10(a). The particularity of their setup lies in the fact that, by creating a Mott insulator phase, exactly one atom is trapped at each node of the lattice and that, despite the relatively small distance between them $(\sim 500 \mathrm{~nm})$, they can be observed independently using a high resolution optical microscope. By sending the excitation laser on all the atoms at the same time, the group observed the production of Rydberg atoms but never closer than the blockade radius, as shown in figure 10(c). They also observed a spatially ordered structure of the Rydberg, as the blockade spheres can not overlap.

\section{Application of the Rydberg blockade to quantum information processing with photons}

Many quantum information protocols, whether in quantum computation or quantum communications, make use of photons. Photons are natural carriers of quantum information as they can travel over long distances and are weakly coupled to the environment, thus limiting their decoherence. There are already dreams of a quantum internet [25], with places where calculations take place are linked by photons to places where information is stored. Many architectures requires the ability to generate photons on demand, the ability to store the information, and the possibility to 
(a)

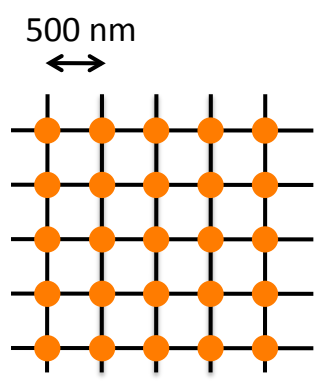

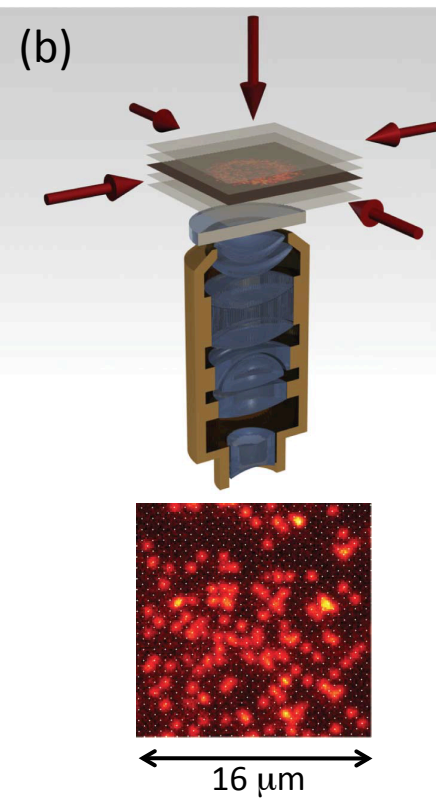

(c)

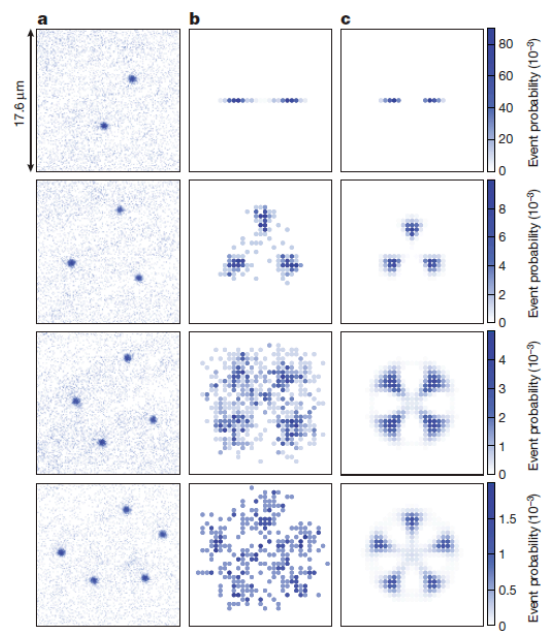

Figure 10: Spatially resolved observation of the blockade. (a) Periodic two-dimensional array of atoms. At each node of the array, a single atom is trapped. (b) High resolution microscope allowing the observation of the individual atoms (from Sherson et al., Nature 467, 68 (2010)). (c) Column a: the dark spot is the signature of a Rydberg atom. Column b: by compiling many pictures such as the ones shown in column a one observes spatially ordered structures, with geometries depending on the number of Rydberg atoms. Column c represents the theoretical predictions. Figures from [24].

create deterministic photonic gates. The Rydberg blockade offers elegant solutions to these requirements, as we now briefly describe.

\subsection{Non-linearity at the single photon level}

In order to create a deterministic photonic gate equivalent to the C-NOT gate described in Section 4.2 for two atoms, two photons have to interact. This is only possible in non-linear media. However traditional media provide non-linearities that are way too weak to be efficient at the level of single photons. This is where the Rydberg blockade comes into play: the idea is to use the strong interaction between Rydberg atoms to create an effective interaction between two photons. This subject of non-linear quantum optics is exploding at the moment, and an introduction by one of the pioneers of this field has recently been published [26].

Besides the Rydberg blockade, the second ingredient to observe strong nonlinearity is the Electromagnetically Induced Transparency (EIT) [27] in a ladder configuration. The principle is illustrated in figure 11(a). Three atomic states $|g\rangle$, $|e\rangle$ and $|r\rangle$ are coupled with a weak probe electromagnetic field (Rabi frequency $\Omega_{\mathrm{p}}$ ) and the strong control field of a laser (Rabi frequency $\Omega_{\mathrm{c}}$ ). If the probe and coupling fields are resonant with the lower and upper atomic transitions respectively, the intermediate state $|e\rangle$ is not populated and the medium is therefore transparent for the probe photons. This comes from the existence of the dark state $\left|\psi_{\text {nc }}\right\rangle \propto$ $\Omega_{\mathrm{c}}|g\rangle-\Omega_{\mathrm{p}}|r\rangle$, not coupled by the lasers to the state $|e\rangle$ and into which the atoms are rapidly optically pumped. If, on the contrary, the coupling laser is not resonant 
(a)

(b)
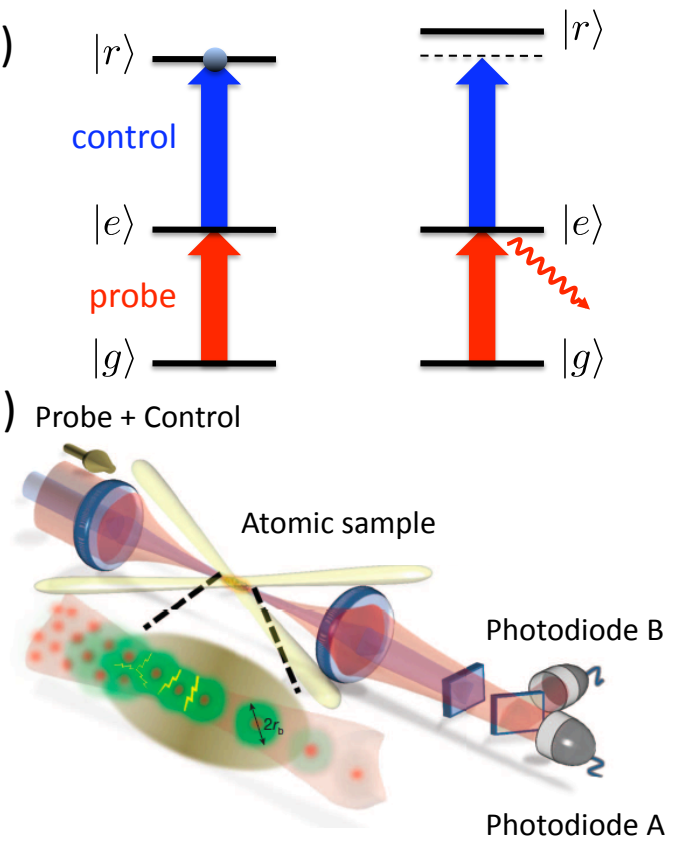

(c)

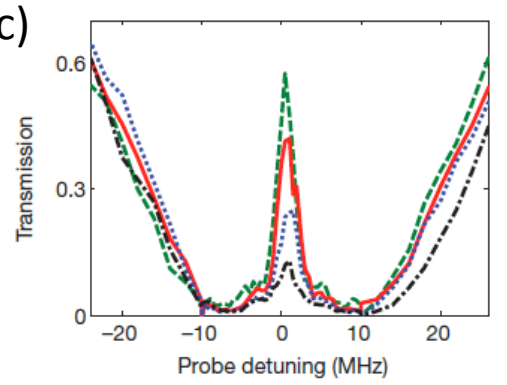

(d)

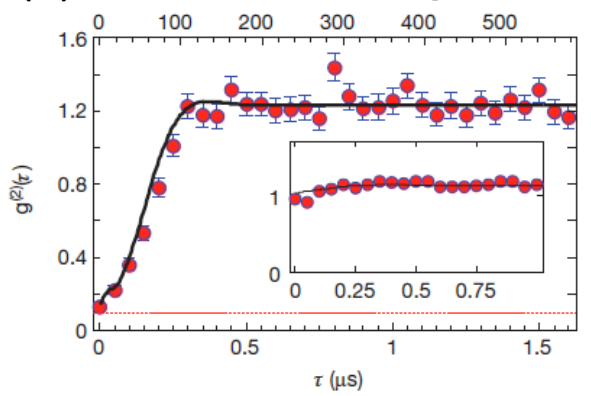

Figure 11: Demonstration of a strong non-linearity at the level of single photons, combining EIT and Rydberg blockade using the high-lying $100 s_{1 / 2}$ state. The figures are extracted from [28]. (a) Principle of the the control of EIT by the Rydberg blockade. (b) Experimental setup. (c) Transmission of the probe field as a function of the detuning with respect to the transition between $|g\rangle$ and $|e\rangle$. The green dotted line corresponds to a low probe photon flux, while the red, blue and black lines corresponds to increasing fluxes. (d) Intensity correlation function. The inset corresponds to the same measurement for the lower, less interacting $46 s_{1 / 2}$ state.

with the upper transition, the photons of the probe field are absorbed by the atoms, which are excited to the state $|e\rangle$.

Combining the blockade and the EIT, the effective interaction between photons can be understood in the simplified following model. We start from an atomic ensemble with a size smaller than the blockade radius, submitted to a control and probe field in an EIT configuration. When a photon from the probe field arrives on the ensemble, one atom gets excited to the Rydberg state and shifts the level of all the other atoms of the sample. The EIT condition is thus no longer fulfilled for the other atoms and a second photon arriving in the medium will be absorbed. The medium therefore filters out multiphoton states of light. As the Rydberg state ultimately decays back to the ground state, the initial photon is sent back in the probe field. The action of the medium is therefore to provide a stream of equally separated photons, which therefore seem to repel each other. The medium is equivalent to a "quantum non-linear filter".

An experiment demonstrating this effect has been performed in 2012 [28]. It opens the way towards photonic gates. The setup is represented in figure 11(b). The control and probe beam are focused in a cold cloud of rubidium atoms held in a dipole trap. The waists of the laser beams are such that the excitation volume is smaller than the blockade radius for the Rydberg state $100 s_{1 / 2}$. The probe beam transmission is measured as a function of its detuning with respect to the transition 
between the states $|g\rangle$ and $|e\rangle$, for different photon fluxes (intensities) of the probe field. The results are shown in figure 11(c): for a low photon flux, the medium is nearly transparent on resonance, while it becomes absorptive at higher fluxes. This is explained by the fact that a large probe field intensity increases the number of Rydberg produced, thus destroying the EIT condition. Figure 11(d) shows the result of the measurement of the intensity correlation function $g^{(2)}(\tau)$ using two detectors. This function characterizes the probability to detect one photon on the first detector and another photon on the second detector after a delay $\tau$. In agreement with the qualitative discussion, the authors of the work observed that $g^{(2)}(0) \approx 0$, meaning that only one probe photon is transmitted at a time through the cloud.

\subsection{Single-photon source and photon storage}

The idea of a single-photon source based on the Rydberg blockade was suggested by M. Saffman in 2002 [29], and demonstrated recently, in particular by the group of C.S. Adams at the university of Durham [30]. The principle is represented in figure 12(a). The excitation of an atomic ensemble with a size smaller than the blockade radius promotes only one atom to the Rydberg state. The subsequent application of the control field only brings the atom in state $|e\rangle$, from where it emits a single photon. One appealing feature comes from the fact that due to the conservation of momentum in this four photon process, the photon is emitted in the direction of the probe field (figure 12b). Figure 12(c) shows the results of an intensity correlation measurement showing that indeed there is a small probability to emit two photons at the same time. The limitation for this particular experiment, explaining that $g^{(2)}(0) \neq 0$, comes from the fact that the size of the atomic sample is not much smaller than the blockade radius.

The group has also demonstrated the principle of a quantum memory by applying a micro-wave field coupling the state $|r\rangle$ to another state $\left|r^{\prime}\right\rangle$ : the atomic excitation is stored in state $\left|r^{\prime}\right\rangle$, where it does not feel the coupling laser. By applying the microwave again after a given storage time, the ensemble can re-emit the photon.

\section{Conclusion}

The field of interacting cold Rydberg atoms is developing very rapidly along several directions: quantum information, quantum optics, the study of quantum many-body systems... Only a few recent experiments along those lines have been discussed in this paper. Despite the quantity of results already obtained, many open questions remain for future work.

For applications in quantum information processing, a major goal is to demonstrate multi-particle entanglement for many atoms. Limits on the size of the entangled systems that can be produced will depend on the results of on-going experiments aiming at improving the fidelity of each manipulation.

Another major avenue concerns quantum simulation, with two main approaches. On the one hand, systems of localized single Rydberg atoms are thought to be an ideal platform to simulate long-range interacting spin systems [31]. On the other hand, it has been proposed to tailor soft-core, long-range interactions between ultracold atoms by using the so-called "Rydberg dressing", i.e. by coupling off-resonantly 

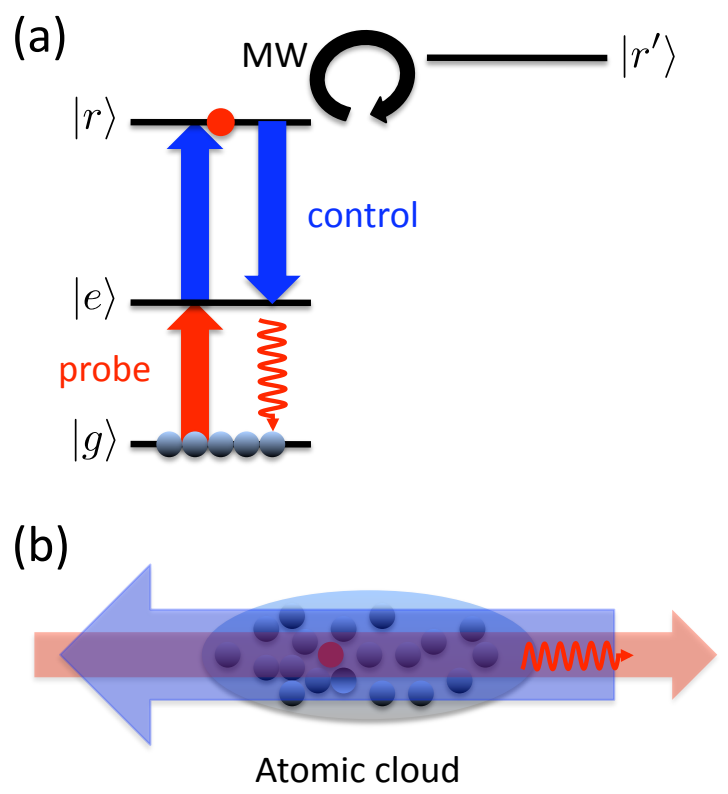

(c)

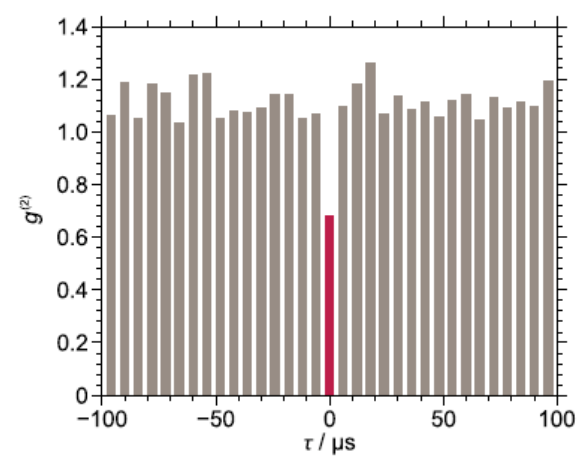

Figure 12: Principle of a single-photon source and storage in an atomic ensemble. (a) Level scheme used. The Rydberg states $|r\rangle$ and $\left|r^{\prime}\right\rangle$ are coupled by a microwave (MW) field. (b) Geometry of the experiment. (c) Result of the measurement of the $g^{(2)}(\tau)$ correlation function (figure from [30]).

the ground state and a Rydberg state. Due to a small admixing of the Rydberg state with the ground state, the atoms experience large, long-range interactions while keeping a long lifetime [32]. New states of quantum matter, with unusual properties, such as supersolids for instance, could then be produced and studied in the laboratory. Finally, as often in research, new, unexpected directions will certainly appear in the future.

\section{References}

[1] T. Gallagher: Rydberg atoms. Cambridge (1994).

[2] N. Bohr: On the constitution of atoms and molecules. Philos. Mag. 26, 1 (1913).

[3] M. Saffman, T. G. Walker, and K. Mølmer: Quantum information with Rydberg atoms. Rev. Mod. Phys. 82, 2313 (2010).

[4] A. Reinhard, T. Cubel Liebisch, B. Knuffman, and G. Raithel: Level shifts of rubidium Rydberg states due to binary interactions. Phys. Rev. 75, 032712 (2007).

[5] J.-M. Raimond, G. Vitrant and S. Haroche: Spectral line broadening due to interaction between very excited atoms: the dense Rydberg gas. J. Phys. B: At. Mol. Phys. 14 L655 (1981).

[6] I. Mourachko, D. Comparat, F. de Tomasi, A. Fioretti, P. Nosbaum, V. Akulin, and P. Pillet: Many-body effects in a frozen Rydberg gas. Phys. Rev. Lett. 80, 253 (1998). 
[7] W.R. Anderson, J.R. Veale, and T.F. Gallagher: Resonant dipole-dipole energy transfer in a nearly frozen Rydberg gas. Phys. Rev. Lett. 80, 249 (1998).

[8] D. Jaksch et al.: Fast Quantum gates for neutral atoms. Phys. Rev. Lett 85, 2208 (2000).

[9] M.D. Lukin et al.: Dipole blockade and Quantum Information in Mesoscopic Atomic Ensembles. Phys. Rev. Lett 87, 037901 (2001).

[10] S. Chu: Nobel Lecture: the manipulation of neutral particles. Rev. Mod. Phys. 70, 685 (1998).

[11] C. Cohen-Tannoudji: Nobel Lecture: Manipulating atoms with photons. Rev. Mod. Phys. 70, 707 (1998).

[12] W. D. Phillips: Nobel Lecture: Laser cooling and trapping of neutral atoms. Rev. Mod. Phys. 70, 721 (1998).

[13] N. Schlosser, G. Reymond, I. Protsenko and P. Grangier: Sub-poissonian loading of single atoms in a microscopic dipole trap. Nature 411, 1024 (2001).

[14] Y. R. P. Sortais et al.: Diffraction-limited optics for single-atom manipulation. Phys. Rev. A 75, 013406 (2007).

[15] E. Urban et al.: Observation of Rydberg blockade between two atoms. Nat. Phys. 5, 110 (2009).

[16] L. Isenhower et al.: Demonstration of a Neutral Atom Controlled-NOT Quantum Gate. Phys. Rev. Lett. 104, 010503 (2010).

[17] A. Gaëtan et al.: Observation of collective excitation of two individual atoms in the Rydberg blockade regime. Nat. Phys. 5, 115 (2009).

[18] T. Wilk et al: Entanglement of Two Individual Neutral Atoms Using Rydberg Blockade. Phys. Rev. Lett. 104, 010502 (2010).

[19] L. Béguin, A. Verner, R. Chicireanu, T. Lahaye, and A. Browaeys: Direct Measurement of the van der Waals Interaction between Two Rydberg Atoms. Phys. Rev. Lett. 110, 263201 (2013).

[20] D. Tong et al.: Local blockade of Rydberg excitation in an ultracold gas. Phys. Rev. Lett. 93, 063001 (2004).

[21] D. Comparat and P. Pillet: Dipole blockade in a cold Rydberg atomic sample. J. Opt. Soc. Am. B 27, A208 (2010).

[22] R. Heidemann et al.: Evidence for collective Rydberg excitation in the strong blockade regime. Phys. Rev. Lett. 99, 163601 (2007).

[23] Y.O. Dudin, L. Li, F. Bariani, and A. Kuzmich: Observation of coherent manybody Rabi oscillations. Nat. Phys. 8, 790 (2012).

[24] P. Schauss et al.: Observation of spatially ordered structures in a twodimensional Rydberg gas. Nature 491, 87 (2012).

[25] H. J. Kimble: The quantum internet. Nature 453, 1023 (2008). 
[26] J.D. Pritchard, K.J. Weatherill, and C.S. Adams: Non-linear optics using cold Rydberg atoms. Annual Review of cold atoms and molecules, 301-350 (2013).

[27] M. Fleischauer, A. Imamoglu and J.P. Marangos: Electromagnetic induced transparency: optics in coherent media. Rev. Mod. Phys. 77, 2003 (2005).

[28] T. Peyronel et al:: Quantum nonlinear optics with single photons enabled by strongly interacting atoms. Nature 488, 57 (2012).

[29] M. Saffman and T.G. Walker: Creating single-atom and single-photon sources from entangled atomic ensembles. Phys. Rev. A 66065403 (2002).

[30] D. Maxwell et al.: Storage and Control of Optical Photons Using Rydberg Polaritons. Phys. Rev. Lett. 110, 103001 (2013).

[31] H. Weimar, M. Müller, I. Lesanovsky, P. Zoller and H.P. Püchler: A Rydberg quantum simulator. Nat. Phys. 6, 382 (2010).

[32] G. Pupillo, A. Micheli, M. Boninsegni, I. Lesanovsky and P. Zoller: Strongly correlated gases of Rydberg dressed atoms: Quantum and classical Dynamics. Phys. Rev. Lett. 104, 223002 (2010). 